\title{
Shared risk factors for atherosclerosis and arteriosclerosis
}

\author{
Claudia Floriana SUCIU1 ${ }^{1}$, Robert Adrian DUMBRAVA², Maria Andrada JIGA², \\ Liviu CRISTESCU ${ }^{2}$, Andreea VARGA ${ }^{1,2}$ \\ ${ }^{1}$ Department ME2, Faculty of Medicine in English, G.E. Palade University of Medicine, Pharmacy, \\ Science and Technology, Targu Mures, Romania \\ 2Internal Medicine - Cardiology II Clinic, Emergency County Hospital, Targu Mures, Romania
}

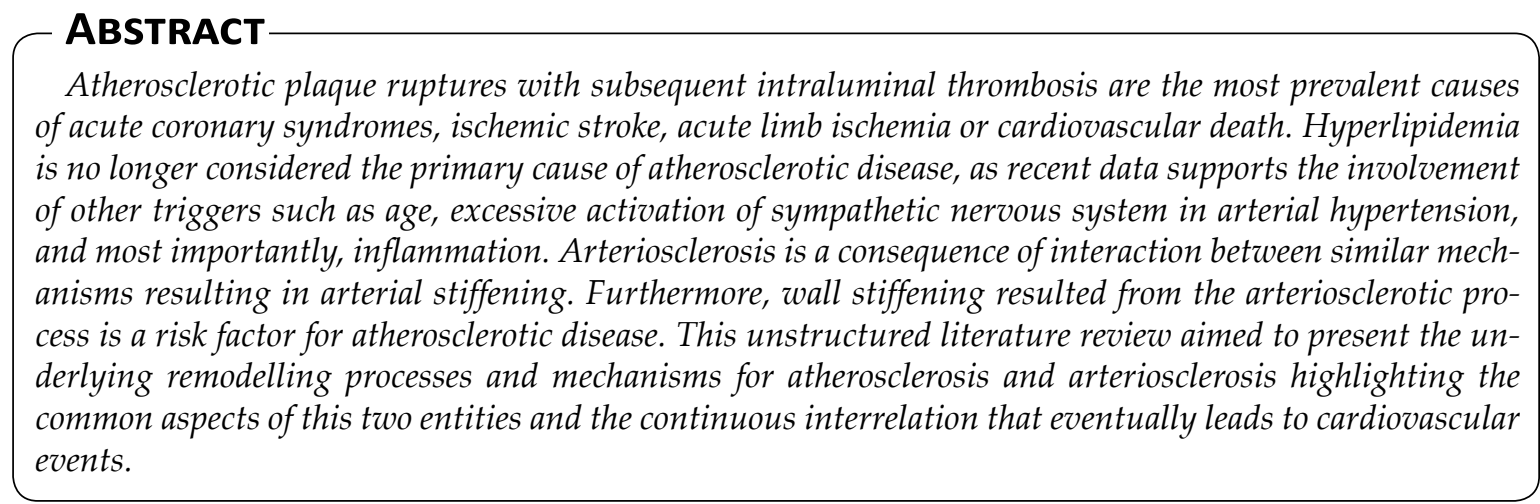

Keywords: atherosclerosis, arteriosclerosis, inflammation, sympathicotonia

\section{INTRODUCTION}

In the last decades important steps forward have been made in understanding the pathogenesis of atherosclerotic disease, thus bringing us closer to the possibility of preventing it's most poignant consequences, namely acute coronary syndromes (ACS), ischemic stroke, acute limb ischemia or cardiovascular death. The most prevalent aforementioned entities are luminal thrombosis following plaque rupture, plaque erosions and calcified nodules.

Arteriosclerosis, an age-related degenerative change of the arterial wall characterized by arterial stiffening without lumen narrowing, represents a distinct process from atherosclerotic disease; however, both pathologies coexist in the same arterial territory and share risk factors such as inflammation. Age-relat- ed chronic inflammatory changes in the arterial wall seem to be the basis of the linking mechanisms between inflammation and arterial stiffness.

\section{ATHEROSCLEROSIS}

Atherosclerotic plaque disruption, superimposed atherothrombosis and subsequent vessel oclussion are responsible for most of the atherosclerotic cardiovascular (CV) events (1). Disruption tends to occur in an plaque with a substantial necrotic core which represents $\geq 30 \%$ of the entire plaque, covered by a thin fibrous cap of less than $65 \mu \mathrm{m}$ (2). Inflammatory cells consisting primarily of macrophages and T-lymphocytes are abundant in these lesions and the matrix metalloproteinases (MMP) released by macrophages 
in the cap, notably MMP- $1,-3$, and -8 , are mainly responsible for the fibrous cap thinning $(3,4)$. However, Johnson et al suggest that MMP-3 and MMP-9 might be involved in plaque stabilization (5).

The rupture of the lesion is preceded by a thin-cap fibroatheroma with a smaller amount of macrophages infiltrating the cap and a smaller necrotic core (4).

An additional characteristic feature for disrupted lesions is the raise of free cholesterols and cholesterol clefts with a decrease in cholesterol esters (6). Cholesterol clefts emerge from cholesterol crystals dissolution by using paraffin for histological preparation. In non-disrupted lesions, esterified cholesterol is the main component of lipid droplets from foam cells as the free cholesterol in excess is still efficiently transported out of the macrophages to HDL particles $(7,8)$.

Negative remodelling of the arterial wall is associated with lumen reduction while positive remodelling of the atherosclerotic arterial wall represents a compensatory outward wall enlargement, that can be favourable provided the plaque load remains $<40 \%$ and the lumen diameter remains constant $(9,10)$. Still, compensatory enlargement of the coronary arterial wall has been associated with rupture prone plaques, as well as with macrophage infiltration in post-mortem examinations $(11,12)$. Additionally, provided circumstances with similar geometrical conditions, coronary arteries with positive remodelling are more prone to rupture when compared with negative remodelling arteries (10).

In contrast to rupture prone plaques, also referred to as vulnerable plaques, the erosion prone plaques showed fewer inflammatory cells and a negative remodelling pattern (11). The erosion prone lesions usually consist in equal measures of the primal atherosclerotic changes namely pathologic intimal thickening and fibroatheroma (2).

The pathologic intimal thickening represents the earliest change and consists of a proteoglycan and collagen matrix with layers of smooth muscle cells rooted by an acellular lipid pool, all accumulated near the lumen (1).

Fibroatheroma is composed of a necrotic core encapsuled by a thick fibrous cap made of smooth muscle cells and a matrix of proteoglycans and collagen (1). Sakakura et al divide fibroatheromas into early and late necrotic core. The early lesions consist of foam cells present at the luminal border of the lipid pools, large cholesterol clefts, signs of extracellular matrix lysis, and presence of proteoglycans, versican, biglycan and hyaluronan, which are typically absent in late necrotic cores. Other characteristic features of late necrotic cores are the presence of free cholesterol, apoptotic macrophages and the absence of a collagenous matrix (2). In both cases the fibrous cap will remain intact and the absence of endothelial lining will be the cause of thrombus formation $(2,13)$.
Even though the primary event leading to atherosclerosis (ASCL) was thought to be represented mainly by hyperlipidemia, new reports support the involvement of other mechanisms such as age, single nucleotide polymorphism, the disruption of repair mechanisms responsible for endothelial wall integrity, sympathetic overactivation encountered in hypertension (HTN) and most importantly, inflammation (14). As a consequence, ASCL is recognized as being characterised by a low-grade chronic inflammation, in which oxidised low density lipoproteins (OxLDL), seen as inflammation by-products, have a significant contribution (15). Subclinical inflammation that occurs with atherosclerotic disease might be an indicator of immune response to the developing changes in the arterial wall during plaque formation, such as endothelial dysfunction and oxLDL generation (15).

Currently observed mechanisms responsible for ASCL report that the innate and adaptive immune system cells accumulate into the atherosclerotic plaque, leading to complex events that result in generation of antibodies against OxLDL and other chronic inflammation by-products. The final results, as concluded in recent data, depend on the lymphocyte subset. Thus, it is suggested that $\mathrm{B} 1$ lymphocyte subsets might have a protective role against $A S C L$, while a pro-atherogenic role is related to $B 2$ lymphocyte subsets (15).

A distinct feature of atherosclerotic disease is represented by vascular calcification. Arterial calcification bears an increased risk for CV morbidity and mortality $(16,17)$. Vascular calcification has a mixed pathomechanism, including intimal calcification specific for atherosclerotic disease, and medial calcification characteristic for arteriosclerosis (18).

\section{ARTERIOSCLEROSIS}

Arteriosclerosis, an age related degenerative change of the arterial wall which occurs in association with ASCL, has also captured a lot of interest when evaluating different etiologies of CV diseases. Characterized by arterial stiffening, it occurs as a consequence of degeneration of extracellular matrix in the media layer of elastic arteries, with loss of elastic fibers and increased fibrosis as a consequence of increased cyclic stress (19). The changes in arterial stiffening are morpho-pathologically different from ASCL, a process that involves the intima layer and is characterized by patchy intimal thickening with subsequent lumen narrowing (1).

Increased arterial stiffness is considered to be the initial indicator of arterial wall dysfunction and, along with traditional $\mathrm{CV}$ risk factors, has an independent predictive value for adverse CV outcomes $(20,21)$. Patients with diabetes mellitus, metabolic syndrome or 
other pathologies with a chronic inflammation status are at high risk of developing increased arterial stiffness $(22,23)$. Furthermore, arterial stiffness is associated with inflammatory markers, such as fibrinogen, $C$ reactive protein (CRP), cytokines, high-sensitive CRP, and also neutrophil-to-lymphocyte ratio, a new emerging inflammatory marker (24). Extensive data regarding inflammation and arteriosclerosis concern CRP, as this inflammatory marker has been reported to be associated with increased arterial stiffness in healthy individuals $(25,26,27)$ and also individuals with known atherosclerotic disease (28).

Additionally, extracellular matrix stiffening from arteriosclerosis might be a risk factor for atherosclerotic disease, as a result of breakage of endothelial barrier. Subsequently, endothelial permeability is increased and cholesterol accumulates in the arterial wall, thus explaining the frequent association between arterial stiffness and plaque (29).

Aside from the direct effect of arteriosclerosis, inflammation as well might represent a common pathological state linking ASCL to arteriosclerosis. Data supporting this hypothesis come from in vivo studies where CRP is reported to decrease nitric oxide synthase (NOS) availability in cultured human aortic endothelial cells (30) with development of increased arterial stiffness as a result of endothelial dysfunction secondary to decline in NOS (31).

Human studies also support inflammation as a possible link between ASCL and arteriosclerosis. The main determinants of increased arterial stiffness are age and increased blood pressure $(32,33)$ while other risk factors having insignificant influence (34). Still, as previously mentioned, arterial stiffening is frequently reported in pro-inflammatory conditions such as diabetes mellitus, insulin resistance, metabolic syndrome regardless of blood pressure or age (22). This occurrence can be justified by arterial wall hypertrophy and fibrosis as a consequence of increased angiotensin type I receptor expression in the context of increased activity of renin-angiotensin-aldosterone system (RAAS). Insulin resistance and hyperinsulinemia are responsible for the stimulation of RAAS $(35,36)$.

Inflammation related to age and CV risk factors enhances arterial stiffness by other means as well, such as metalloproteinases synthesis up-regulation, which leads toward an imbalance between elastin production and breakdown/degradation of basement membranes (37).

Moreover, the age-related chronic pro-inflammatory profile bears a specific senescence phenotype, characterized by an increase in inflammatory modulators, such as MCP-1, and cytokines (IL-1, IL-6, IL-17) (38) and also increased activity of RAAS which results in an increased vascular smooth muscle tone with age-related arterial remodelling (39).
The sympathetic nervous system is involved in regulating average blood pressure and resistance of vascular system. Changes in the normal activity of the sympathetic nervous system may be responsible for idiopathic HTN (40). Hypertensive patients present decreased baroreceptor sensitivity with an increased sympathetic activity and reduced parasympathetic tone compared with normotensive controls (41). In HTN, a permanent increase in mean blood pressure associated with increased sympathetic activity induce changes in the elastic properties of the arterial wall, resulting a permanent reduction of arterial distensibility and increased stiffness (42). As a consequence, hypersympatheticotonia may be another common risk factor for arteriosclerosis and ASCL, due to the direct effect on the vascular wall and also as a consequence of the inflammation induced by granulocytosis secondary to local vascular activation of sympathetic nervous systems. In addition, changes in mean blood pressure, heart rate and left ventricular function, can also be considered as determinant factors of transient changes in elastic arterial properties $(43,44)$.

A clear distinction between the different stages of atherosclerotic disease is impossible without refined imaging studies. In the absence of suggestive symptomatology or classic risk factors for atherosclerotic disease, patients do not present an indication for comprehensive investigations. Taking in consideration recent studies reporting that classic risk factors no longer account for all cases of atherosclerotic disease, novel risk factors for ASCL and arteriosclerosis, such as inflammation might aid in selecting asymptomatic individuals without obvious risk factors for further multimodality imaging studies.

Arteriosclerosis gained recognition in recent years as an emerging entity with important implications in CV morbidity and mortality. Proper evaluation requires well-trained personell and inaccessible or hard to reach tools. Furthermore, a clear consensus on appropriate evaluation of arteriosclerosis is still lacking (45). Inflammation however, proved to be a reliable predictor for arteriosclerosis, hence, it can aid in selecting individuals with risk for arterial stiffness as well.

\section{CONCLUSIONS}

Both atherosclerosis and arteriosclerosis are important factors of CV diseases. Even though these are distinct afflictions of the arterial wall, they present morphopatologically interrelated processes. Furthermore, they share important, conclusive risk factors such as chronic inflammation, age and hypersympatheticotonia.

\section{Acknowledgement}

All authors have contributed to the published version of the manuscript. 


\section{REFERENCES}

1. Virmani R, Kolodgie FD, Burke AP et al. Lessons from sudden coronary death: $A$ comprehensive morphological classification scheme for atherosclerotic lesions. Arterioscler Thromb Vasc Biol. 2000;20:1262-75.

2. Sakakura K, Nahano M, Otsuka F et al. Pathophysiology of atherosclerosis plaque progression. Heart Lung Circ. 2013;22:399411.

3. Sukhova GK, Schonbeck U, Rabkin E et al. Evidence for increased collagenolysis by interstitial collagenases- 1 and -3 in vulnerable human atheromatous plaques. Circulation. 1999;99:2503-9.

4. Newby AC. Metalloproteinase expression in monocytes and macrophages and its relationship to atherosclerotic plaque instability. Arterioscler Thromb Vasc Biol. 2008;28:2108-14.

5. Johnson JL, Dwivedi A, Somerville M et al. Matrix metalloproteinase (MMP)-3 activates MMP-9 mediated vascular smooth muscle cell migration and neointima formation in mice. Arterioscler Thromb Vasc Biol. 2011;31:e35-44.

6. Felton CV, Crook D, Davies MJ, Oliver MF. Relation of plaque lipid composition and morphology to the stability of human aortic plaques. Arterioscler Thromb Vasc Biol. 1997:17:1337-45.

7. Li AC, Glass CK. The macrophage foam cell as a target for therapeutic intervention. Nat Med. 2002;8:1235-42.

8. Rhainds $D$, Brissette $L$. The role of scavenger receptor class B type I (SR-BI) in lipid trafficking. Defining the rules for lipid traders. Int J Biochem Cell Biol. 2004;36:39-77.

9. Glagov S, Weisenberg E, Zarins CK et al. Compensatory enlargement of human atherosclerotic coronary arteries. $N$ Engl J Med. 1987;316:1371-5.

10. Cilla M, Peña E, Martínez MA, Kelly DJ. Comparison of the vulnerability risk for positive versus negative atheroma plaque morphology. J Biomech. 2013;46:1248-54.

11. Burke AP, Kolodgie FD, Farb A et al. Morphological predictors of arterial remodeling in coronary atherosclerosis. Circulation. 2002;105:297-303.

12. Varnava AM, Mills PG, Davies MJ. Relationship between coronary artery remodelling and plaque vulnerability. Circulation. 2002;105:939-43.

13. Tabas I. Cholesterol and phospholipid metabolism in macrophages. Biochim Biophys Acta. 2000;1529:164-74.

14. Head T, Daunert S, Goldschmidt-Clermont PJ. The aging risk and atherosclerosis: A fresh look at arterial homeostasis. Front Genet. 2017;8:216.

15. Suciu CF, Prete M, Ruscitti P et al. Oxidized low density lipoproteins: The bridge between atherosclerosis and autoimmunity. Possible implications in accelerated atherosclerosis and for immune intervention in autoimmune rheumatic disorders. Autoimmun Rev. 2018;17:366-75.
16. Iribarren C, Sidney S, Sternfeld B, Browner WS. Calcification of the aortic arch: risk factors and association with coronary heart disease, stroke, and peripheral vascular disease. JAMA. 2000;283:2810-5.

17. Li Y, Chen X, Huang L, Lu J. Association between neutrophil-lymphocyte ratio and arterial stiffness in patients with acute coronary syndrome. Biosci Rep. 2019;39:BSR20190015.

18. Takasu J, Katz R, Nasir K et al. Relationships of thoracic aortic wall calcification to cardiovascular risk factors: the Multi-Ethnic Study of Atherosclerosis (MESA). Am Heart J. 2008;155:765-71.

19. Lee HY, Oh BH. Aging and arterial stiffness. Circ J. 2010;74:2257-62.

20. Cavalcante JL, Lima JA, Redheuil A, Al-Mallah MH. Aortic stiffness: current understanding and future directions. J Am Coll Cardiol. 2011;57:1511-22.

21. Vlachopoulos C, Aznaouridis K, Stefanadis C. Prediction of cardiovascular events and all-cause mortality with arterial stiffness: a systematic review and meta-analysis. J Am Coll Cardiol. 2010;55:1318-27.

22. Stehouwer CD, Henry RM, Ferreira I. Arterial stiffness in diabetes and the metabolic syndrome: a pathway to cardiovascular disease. Diabetologia. 2008;51:527-39.

23. Kinlay S, Creager MA, Fukumoto $M$ et al. Endothelium-derived nitric oxide regulates arterial elasticity in human arteries in vivo. Hypertension. 2001;38:1049-53.

24. Mozos I, Malainer C, Horbańczuk J et al. Inflammatory markers for arterial stiffness in cardiovascular diseases. Front Immunol. 2017;8:1058.

25. Yasmin, McEniery CM, Wallace $S$ et al. C reactive protein is associated with arterial stiffness in apparently healthy individuals. Arterioscler Thromb Vasc Biol. 2004:24:969-74.

26. Okamura T, Moriyama Y, Kadowaki T et al. Non-invasive measurement of brachialankle pulse wave velocity is associated with serum $C$ reactive protein but not with alpha-tocopherol in Japanese middle-aged male workers. Hypertens Res. 2004:27:173-80.

27. Mattace-Raso FU, van der Cammen TJM, van der Meer IM et al. C-reactive protein and arterial stiffness in older adults: the Rotterdam Study. Atherosclerosis. 2004;176:111-6.

28. Makita S, Nakamura M, Hiramori K. The association of C-reactive protein levels with carotid intima-media complex thickness and plaque formation in the general population. Stroke. 2005;36:2138-42.

29. Huynh J, Nishimura N, Rana K et al. Age-related intimal stiffening enhanced endothelial permeability and leukocyte transmigration. Sci Trans/ Med. 2011:3:112ra122.

30. Venugopal SK, Devaraj S, Yuhanna I et al. Demonstration that $\mathrm{C}$ reactive protein decreases eNOS expression and bioactivity in human aortic endothelial cells.

Circulation. 2002;106:1439-41.

31. Kinley S, Creager MA, Fukumoto $M$ et al. Endothelium-derived nitric oxide regulates arterial elasticity in human arteries in vivo. Hypertension. 2001;38:1049-53.

32. Kozakova M, Morizzo C, Guarino D et al. The impact of age and risk factors on carotid and carotid-femoral pulse wave velocity. J Hypertens. 2015;33:1446-51.

33. Al Ghatrif M, Strait JB, Morrell $\mathrm{CH}$ et al. Longitudinal trajectories of arterial stiffness and role of blood pressure. The Baltimore Longitudinal Study of Aging. Hypertension. 2013;62:934-41.

34. Cecelja M, Chowienczyk P. Dissociation of aortic pulse wave velocity with risk factors other than hypertension. A systematic review. Hypertension. 2009;54:1328-36.

35. Brillante DG, O'Sullivan AJ, Howes LG. Arterial stiffness in insulin resistance: the role of nitric oxide and angiotensin II receptors. Vasc Health Risk Manag. 2009;5:73-8.

36. Jesmin S, Sakuma I, Hattori Y, Kitabatake A. Role of angiotensin II in altered expression of molecules responsible for coronary matrix remodeling in insulin resistant diabetic rats. Arterioscler Thromb Vasc Biol. 2003;23:2021-6.

37. Park S, Lakatta EG. Role of inflammation in the pathogenesis of arterial stiffness. Yonsei Med J. 2012;53:258-61.

38. Accardi G, Aiello A, Gambino CM et al. Mediterranean nutraceutical foods: strategy to improve vascular ageing. Mech Ageing Dev. 2016;159:63-70.

39. Wang M, Jiang L, Monticone RE, Lakatta EG. Proinflammation: the key to arterial aging. Trends Endocrinol Metab. 2014;25:72-9.

40. Charkoudian N, Rabbitts JA. Sympathetic neural mechanisms in human cardiovascular health and disease. Mayo Clin Proc. 2009;84:822-30.

41. Guzzetti S, Piccaluga E, Casati R et al. Sympathetic predominance in essential hypertension: a study employing spectral analysis of heart rate variability. $J$ Hypertens. 1988;6:711-7.

42. Bruno RM, Ghiadoni L, Seravalle $G$ et al. Sympathetic regulation of vascular function in health and disease. Front Physiol. 2012;3:284.

43. Lantelme $P$, Mestre $C$, Lievre $M$ et al. Heart rate: an important confounder of pulse wave velocity assessment. Hypertension. 2002;39:1083-7.

44. Salvi P, Palombo C, Salvi GM et al. Left ventricular ejection time, not heart rate, is an independent correlate of aortic pulse wave velocity. J Appl Physiol. 2013;115:1610-7.

45. Kim HL, Kim SH. Pulse Wave Velocity in Atherosclerosis. Front Cardiovasc Med. 2019;6:41. 